\title{
AN APPROACH FOR COMMUNICATION RELIABILITY USING SELF-ADAPTIVE AUTONOMIC COMPUTING TECHNIQUES
}

\author{
$U D C(621.311 .3:(007: 621.391: 004))$
}

\begin{abstract}
Aleksandar Stanimirović ${ }^{1}$, Miloš Bogdanović ${ }^{1}$, Nikola Davidović ${ }^{1}$, Aleksandar Dimov ${ }^{2}$, Krasimir Baylov², Leonid Stoimenov ${ }^{1}$

${ }^{1}$ University of Niš, Faculty of Electronic Engineering, Computer Science Department, Niš, Republic of Serbia

${ }^{2}$ University of Sofia, Faculty of Mathematics and Informatics, Department of Software Engineering, Sofia, Republic of Bulgaria
\end{abstract}

\begin{abstract}
Interdependency of electric power grids and information and communication technology is a rapidly growing topic. With the introduction of Smart Grid, handling dynamic load tracking, dynamic tarifs, clients that can consume but also produce electricity that can be delivered to the grid has become a part of everyday operational cycles within power supply companies. Hence, electricity distribution and power supply companies are in need for introduction of efficient mechanisms for the optimal tracking and use of available electric energy. In this paper, we describe the low voltage $(L V)$ distribution network monitoring system developed for the Electric Power Industry of Serbia (EPS) electricity distribution company. The system we present is implemented in a way so that it provides abilities to measures, communicates and stores real-time data, translating it into actionable information needed by EPS to meet the described challenges regarding $L V$ distribution networks. The implemented system is using selfadaptive autonomic computing techniques to provide a reliable data transfer from measurement devices deployed in different parts of the LV distribution network.
\end{abstract}

Key words: self-adaptive, autonomic computing, low voltage network monitoring, communication reliability, smart grid

Received March 26, 2019

Corresponding author: Aleksandar Stanimirović

Faculty of Electronic Engineering, Computer Science Department, Niš, Republic of Serbia

E-mail: aleksandar.stanimirovic@elfak.ni.ac.rs 


\section{INTRODUCTION}

With the introduction of Smart Grid [1], interdependency of electric power grid and information and communication technology is rapidly emerging [2]. This means that not only are electric power grid devices shifting from the analogue technology to digital, which in turns provides more possibility of data measurement and control, but it also means that these devices are starting to be interconnected.

But Smart Grid also means that more stakeholders are involved into the power generation and distribution process, hence more data are being gathered and more diversified analyses of such data are needed. Nowadays, dynamic load tracking, dynamic tariffs, clients that can consume but also produce electricity that can be delivered to the grid are becoming a reality [3]. Efficient use of electric energy necessitates the introduction of efficient mechanisms for the optimal use of available electric energy.

An electricity distribution system includes a complex power supply network in the form of an electrical grid which consists of a huge number of power lines and devices. Monitoring and management of such a complex network represents a major challenge in the daily work of companies for electricity distribution and power supply. These companies have realized that information gathered from sensors and specialized measuring devices are gaining more importance in creating successful business plans. The efficient use of data provided from sensors and measuring devices can lead to reducing cost and delivering better services to the customers. But in order to be able to process such high volumes of data, conventional approaches cannot be used anymore. If such data are only stored into the data warehouse and analysed later, it will not have the same impact as if the analyses are obtained in real-time or in near real time. One of the possible approaches is to use stream computing systems to be able to process such high volumes of data as they come.

Most solutions that can be found in the literature are focusing on high and medium voltage network automation and smart meters that are possible to be read automatically. In most cases a deep investigation of the low voltage (LV) network has been left disregarded, even if it represents the asset bridging the high and medium voltage levels up to final customers. The LV network segment is probably the most affected one by international regulatory changes that are promoting renewable energy sources, as a way to reduce greenhouse gas emissions, diversify the energy supply, diminish the dependence on imported fuels and, more in general, allow the transition to more sustainable energy paradigms [4]. Thus, monitoring the real operating conditions of the LV networks in terms of power flows, phase imbalances, voltage levels and other power quality indicators becomes essential to efficiently operate these kinds of networks. In terms of effectively managing the LV distribution network and the challenges presented by both electricity losses and renewable technologies, electric distribution companies need access to accurate, timely data and actionable information.

This paper describes the LV monitoring system developed for the Electric Power Industry of Serbia (EPS) electricity distribution company. The developed system measures, communicates and stores real-time data, translating it into actionable information needed by EPS to meet described challenges regarding LV distribution networks. Different sensors have been used to collect near real-time data (among which current, voltage, power and energy values) about medium voltage to low voltage electrical 
substations in distribution network. The data this system collects is transmitted to its data centre, where it is analyzed and presented as situational analysis of the LV distribution network conditions. Analysis tools provide daily load profiles and voltage level data to help company create plans for 'stress points', energy losses in the network and maintain statutory voltage levels. The presented system devotes a special attention to reliability and completeness of the data it gathers. For this reason, it is enhanced with self-adaptation features capable of decreasing the number of data retrieval failed communications. As a result, this system handles measurement devices differently depending on the current system state and decreases the number of failed communications retries.

The remainder of this paper is organized as follows. Section II reviews previous works regarding monitoring LV electricity distribution network and using autonomous computing and self-adaptation systems. Section III presents basic theoretical background about autonomous computing. Section IV presents the system designed and deployed for monitoring LV distribution network within EPS electricity distribution company. Section V presents different approaches used by the LV electricity distribution network system to provide a reliable communication with measurement devices. Finally, Section VI provides concluding remarks.

\section{RELATED WORK}

Significant advancement in sensor technology developed many opportunities for involving sensors in numerous industrial systems. Along with its numerous uses, sensor technology found its place in power grids. Here, electricity distribution companies found sensors to be very useful for monitoring and analysing electrical grids. Sensors became a part of the systems responsible for monitoring the state of electrical grid and predicting its behaviour based on the data they collect.

Different solutions and systems already applied in transmission networks have been progressively integrated at the high and medium voltage levels of distribution networks. Fault Location, Isolation and Service Restoration (FLISR) technologies, that have gained sustained attention in the past few years, are a typical example of such solutions. FLISR solutions have been investigated from both a theoretical and a practical viewpoint and they are now at a deployment stage $[5,6]$. Further, smart metering initiatives have been promoted as a way to improve customers relationship management and to foster their awareness on their energy habits [7]. These technologies are designed not just to reduce customers' bills, to save energy or to improve customers comfort, but also to use energy in a more efficient way by means, for example, of load shifting to off-peak hours, demand adaptation to renewable sources supply, reactions to emergency conditions [8].

Grilo et al. have explored the approach of using wireless sensor and actuator networks for improving electrical power grid reliability within the Wireless Sensor and Actuator Networks for Critical Infrastructure Protection (WSAN4CIP) project [9]. In order to improve the grid's safety and reliability of the substations' components, they have used cameras equipped with infrared thermal sensors, temperature sensors and light actuators. They have integrated this system with an existing SCADA system in order to provide a unified interface for operators and a trail testing is organized in the power distribution company in Portugal. 
Another research project, described by Leon et al., was focused on the utilization of wireless sensor networks for detecting mechanical failures in transmission lines [10]. This approach uses sensors in order to successfully determine whether the hazard happened due to the environment- (wind, snow, ice, flood, etc.) or is human-related (accidents, terrorism). For solving this problem, they have deployed and utilized tension, displacement, acceleration and temperature sensors, which were installed in transmission line towers in order to communicate wirelessly with control centres. The system recognizes a hazardous situation based on predefined values for each case and is able to diagnose electrical faults and determine measures that could be automatically taken. Further, the proposed system can provide a complete physical and electrical picture and an alert if an extreme mechanical condition is identified on a transmission line. Also, there was a research conveyed by the Mississippi State University that was related to wide area monitoring of an electrical system and its integration within the existing SensorNet management system [11]. The motivation for this research was the noticeable increase in frequency of blackouts during the last decade.

As monitoring capabilities grew, approaches based on stream computing and stream mining of real-time or near-real-time data gained importance. These approaches have been used for quite some time in various specific fields such as monitoring of the highway traffic [12]. But also, there are generic approaches with platforms that can be customized for the real-life deployments, such as S4 [13]. Only lately, after the Smart Grid development has influenced such high data volumes being obtained [14], it is starting to be used in the field of EPD. In [15] authors are identifying the most important technologies that are used in Smart Grids, namely: Streaming Data Analytics, Load Forecasting, Cloud, Demand Response and Static Analytics. Obviously, first three and the fifth require streaming computing of incoming data to make the system adaptable to dynamical changes that happen throughout the grid. Predicting power supply and demand is one of the big challenges that can be solved using Stream Computing [16]. But not only academia is trying to give answers to such demands for the real-time data analysis, companies like Accenture are already providing solutions that can fulfil such needs [17].

There are many existing works in the field of self-adaptation. For example, MORPH [18] is reference architecture for configuration and behaviour self-adaptation. It allows adaptation of system configurations and behaviour in an independent and coordinated way. MORPH emphasizes on having reconfiguration and behaviour control as first-class architecture entities. It is structured in three main layers: Goal Management, Strategy Management and Strategy Enactment. They all share a common Knowledge Repository. All of the presented layers are based on the MAPE-K loop. However, they have different responsibilities. The Goal Management layer is responsible for setting and adjusting the overall goals for the entire system. The Strategy Management layer is responsible for the adaptation based on a set of predefined strategies. Once the most suitable strategy is selected, the Strategy Enactment layer executes it.

Rainbow $[19,20]$ is a framework that allows engineering software systems with selfadaptive capabilities at runtime. It provides mechanisms for monitoring target systems, detecting opportunities for adaptation, deciding what actions to take, and implementing those actions. Rainbow uses external adaptation mechanisms, which allows developers to specify adaptation strategies for multiple characteristics of the systems. The framework uses architecture-based self-adaptation [20]. An architectural model represents the architecture of 
the entire system as a graph of components that interact with each other. Rainbow consists of three main reusable units - system-layer, architecture-layer, and translation.

\section{AUTONOMIC COMPUTING}

Autonomic computing has been introduced about a decade ago as an attempt to define structural approach towards design and development of dynamic and self-adaptive software systems.

Self-adaptive systems can be viewed from multiple points depending on their direction of autonomy. These points are called self-* properties of the system. They represent the ability of the system to take autonomous actions in the following 4 areas [21]:

- Self-configuration - the system is able to configure itself in order to comply with initially defined high level goals;

- Self-optimization - the system is able to detect and optimize weak points or places that can be improved in terms of specific requirements;

- Self-healing - the system is able to detect problems, create a strategy for fixing them and finally - to apply it;

- Self-protection - the system is able to detect potential threats and defend itself against external attacks.

The reference architecture designed here should propose an approach that will target the Self-configuration and Self-optimization properties of the distributed system. The idea behind this is that self-adaptive systems can dynamically change their behaviour or properties based on external stimulus. A key mechanism used for running the selfadaptation is the so-called control loop or feedback loop [22]. It contains the following main phases (Fig. 1):

- Collect - the relevant data is obtained from the surrounding environment and stored for further processing. Usually, this data is collected through agents or sensors.

- Analyse - the data is analysed and related to existing models.

- Decide - the best action from a list of options is selected

- $\boldsymbol{A c t}$ - the system puts the change in place and the loop starts over.

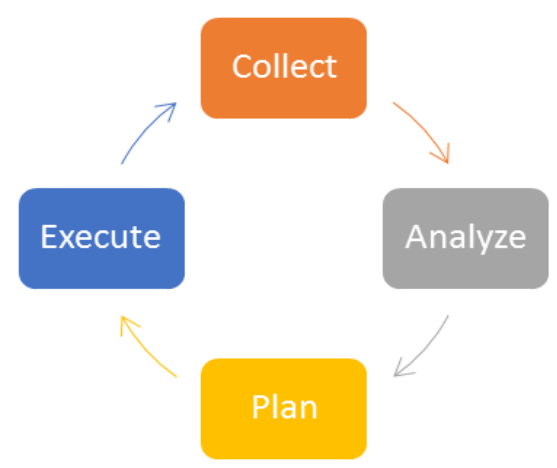

Fig. 1 Autonomic control loop 


\section{LOW Voltage EleCtricity Distribution NETWORK MONITORING SYSTEM}

Currently there are several initiatives for monitoring LV different segments of electricity distribution network owned by the Electric Power Industry of Serbia (EPS) electricity distribution company. EPS is a state-owned electric utility power company and at the moment de facto holds a monopoly on the electricity market in Serbia. One of the main problems of EPS is efficient monitoring of delivered electric energy and charging customers for the delivered energy on time and without errors. At the moment, only small fraction of all EPS customers is covered with smart meters and the Advanced Metering Infrastructure (AMI). However, the process of replacing old analogue meters with modern smart meters and AMI has already been taking more than a decade and will probably continue for a significant period of time in the foreseeable future. In a such situation, EPS cannot rely on using smart meters and AMI for LV distribution network monitoring. In order to overcome this problem and to provide means for LV distribution network monitoring, EPS choose a different approach. For a few years already, the company is deploying various measuring devices, grid analyzers and smart sensors within LV network. These devices are used for remote monitoring of LV network conditions: voltage level, currency strength, delivered energy, etc.

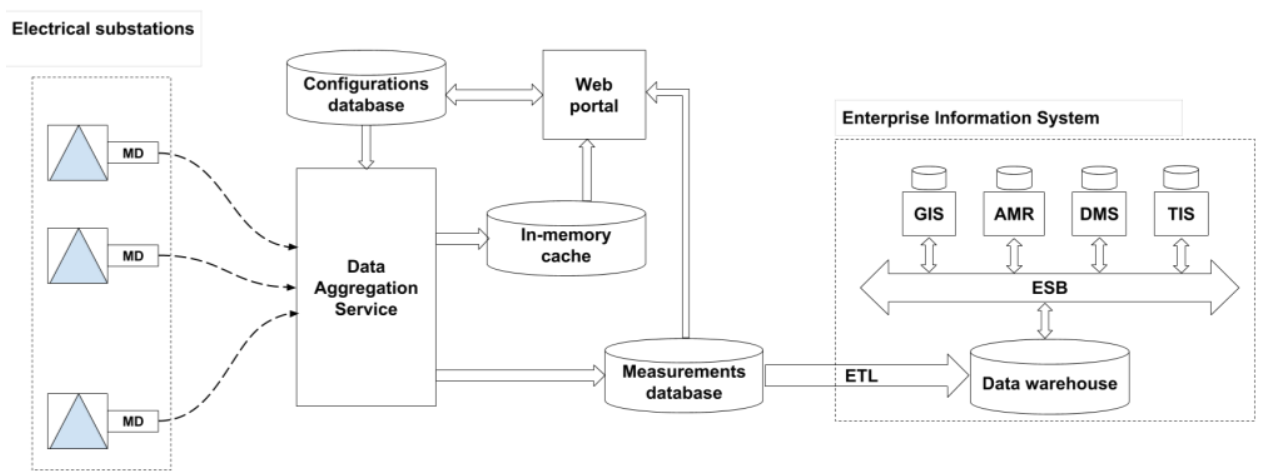

Fig. 2 Low voltage network monitoring system

The paper describes the LV distribution network monitoring system developed for the EPS. This system is based on using smart measuring devices to monitor different LV distribution network segments, mostly middle voltage to low voltage electrical substations and parts of LV distribution network between these electrical substations and meters on the customer side. The developed and deployed system collects measured data from smart sensors, transmits the collected data to a data centre and stores near real-time data, translating it into actionable information to provide EPS with better understanding on conditions in different segments of the LV distribution network.

Architecture of the deployed systems for the LV electricity distribution network monitoring consists of several components (Fig. 2):

- Measuring devices (MD) are smart sensor devices deployed in middle voltage to low voltage electrical substations or different parts of the low voltage distribution network.

- Data Aggregation Service (DAS) is responsible for gathering sensor data from MD 
- Web portal as administrative and supporting tool for Data Aggregation Service and reporting tool for collected sensor data.

- Configurations database (CDB) for keeping DAS configuration data.

- Measurements database (MDB) for storing collected measurements from sensors.

- In-memory cache (IM) for temporary storing of most current readings collected from sensors.

- File storage (FS) for storing collected sensor data in raw format.

- Data Warehouse (DW) for permanent storing of sensor data after cleansing and transformation process.

- Enterprise information system (EIS) (based on existing integration platform [23]).

\section{Measuring devices}

There are many electrical substations throughout the utility power grid. The proposed architecture provides for the possibility that each electrical substation is equipped with one or several measuring devices. Such a measuring device is equipped with built-in sensors for monitoring of electrical energy consumption, power quality analysis, and management of electrical energy use.

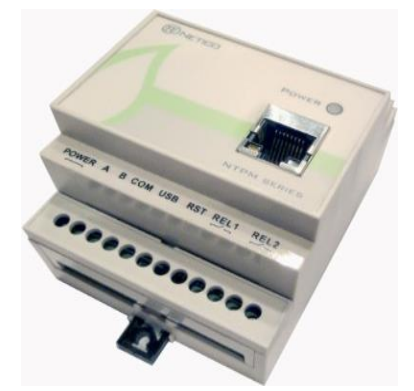

a)

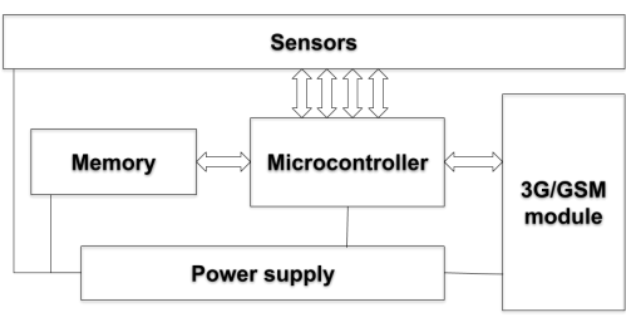

b)

Fig. 3 Energy management sensor a) sensor case b) sensor schematic

Our system uses an MCU based power quality analyzer unit installed in the field and it measures the power supply quality on a $10 \mathrm{kV} / 0.4 \mathrm{kV}$ network points (Fig. 3) [24]. This energy measurement sensor supports measurement and automatic calculation of more than 100 electrical energy parameters including: power grid frequency, root mean square voltage, root mean square current, active power, reactive power, apparent power, power factor of each phase, total active power, total reactive power, total apparent power, total power factor, total active energy and others. Each energy measurement sensor is equipped with solid state memory and supports the permanent storage of measured and calculated parameters. A history of all measured data is stored with 1-second resolution for a period of 1 month. In addition, the device stores up to 10 years history of the most important energy parameters calculated on hourly bases (1-hour resolution).

Communication between energy measuring devices and the system data centre is based on usage of GSM networks (e.g. GPRS/UMTS/3G). Data centre and all measurement devices are 
a part of the same virtual network and every device is accessible on that network as an HTTP endpoint. Our system collects data from measurement devices by sending HTTP POST requests and receiving and reading appropriate responses.

On Fig. 4 an example of a properly formed request is shown. The request data is sent using the form-urlencoded media type, the Content-Type header value must be set to "application/x-www-form-urlencoded". The request data contains different parameters that are sent to a measurement device specifying which measurements to read. In this example, 15 minutes measurements are requested for a 24-hour period. The tags parameter specifies measurement values that are requested from the device.

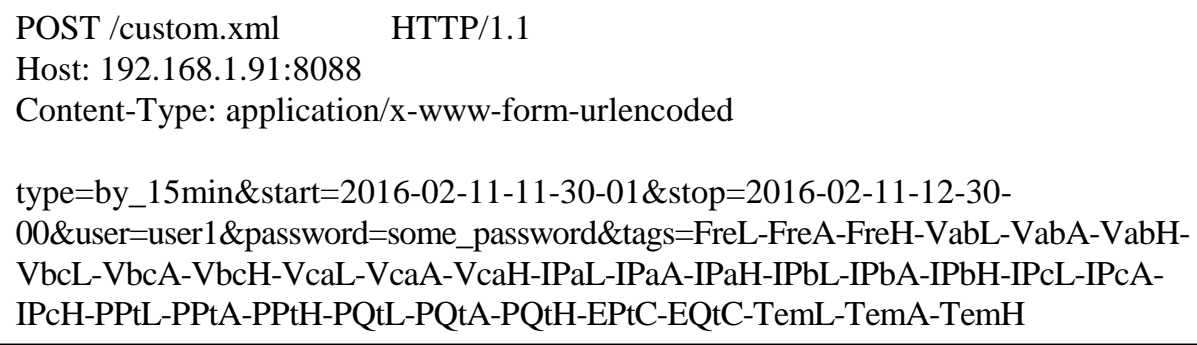

Fig. 4 Properly formed HTTP POST request to obtain data from energy measurement device

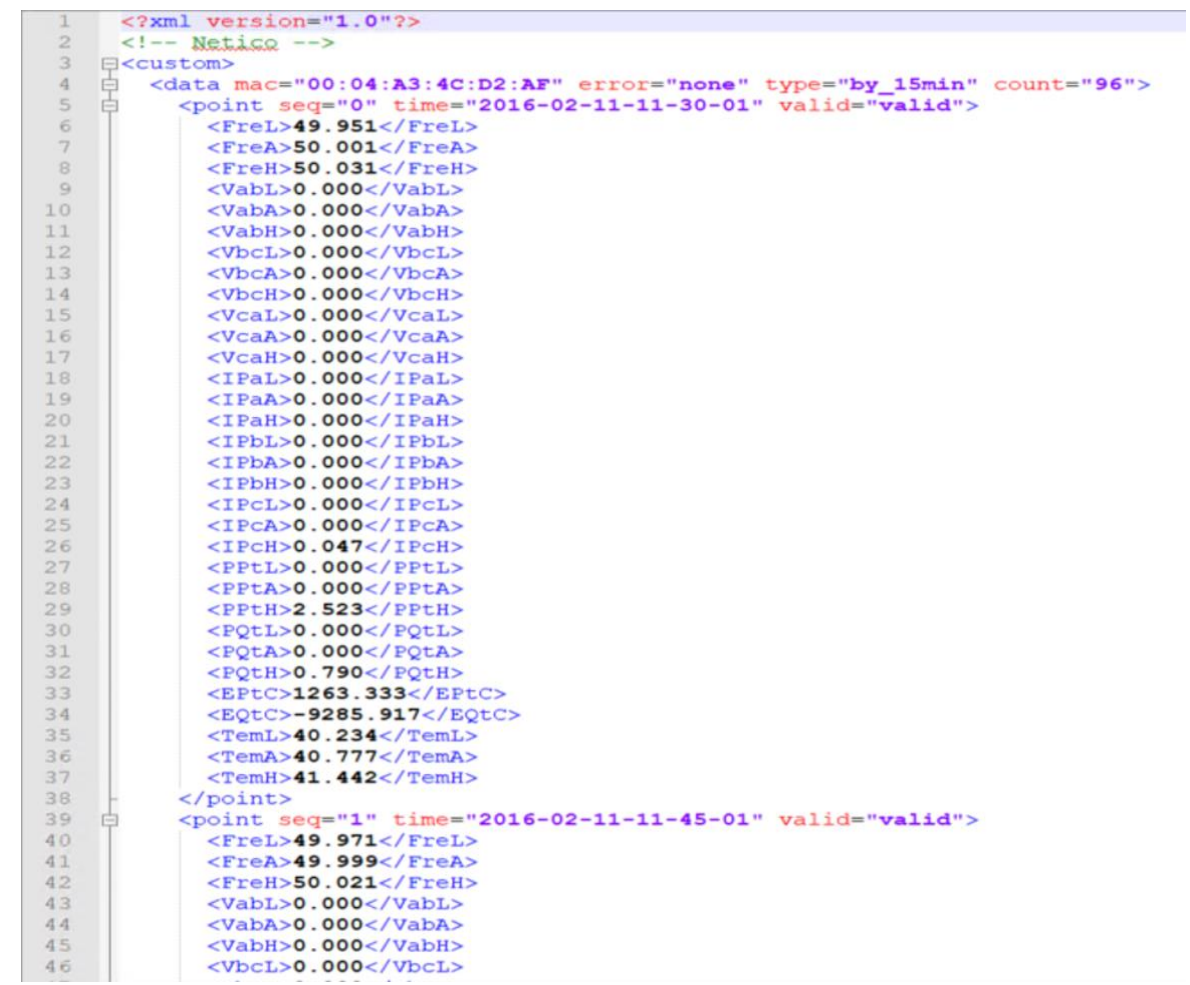

Fig. 5 Example of a valid response from energy measurement device 
Fig. 5 presents an example of a valid response from energy measurement device (response is obtained using request from Figure 3). Response is received as a properly formed XML file. This XML file contains requested measurements from a device in the form of a collection of data points. Each data point represents a measurement in a specific moment of time. In this example, 15 minutes measurements are requested for a 24-hour period and XML document contains 96 data points. Each data point contains values for all requested tags.

\section{Data Aggregation Service}

The Data Aggregation Service (DAS) is responsible for gathering information from the electrical substations (precisely, from measuring devices in electrical substations). The service implements a task scheduler system. Every task in this system represents an action of polling measurements data from the measuring devices using GSM network.

DAS collects measurements data from electrical substations in two different regimes:

- Current measurements - represents the current status of an electrical substation. These measurements are usually read with a 5-minutes frequency and stored into an in-memory cache. There is no need for store keeping these data because measurements are replaced with new values in every reading cycle.

- History measurements - represents average values for a given period of time (usually we are dealing with a 15-minutes measurements). The service pools this data from electrical substations with different frequencies: usually every 15 minutes (one measurement) or every hour (four measurements in a single read request). History measurements, received from electrical substations, are extracted from a received XML file and stored in measurements database without further processing. Usually some extract-transform-load process is defined to validate, and process collected data and to load that data into the company data warehouse. Also, if necessary, original XML files can be stored in a raw format in file storage system for later processing.

\section{Web portal}

Web portal is an important part of the system and provides users with a quick and easy way to:

- Control DAS settings,

- Schedule tasks for reading measurements data, and

- Create reports based on measurements data that DAS collects from measuring devices in electrical substations.

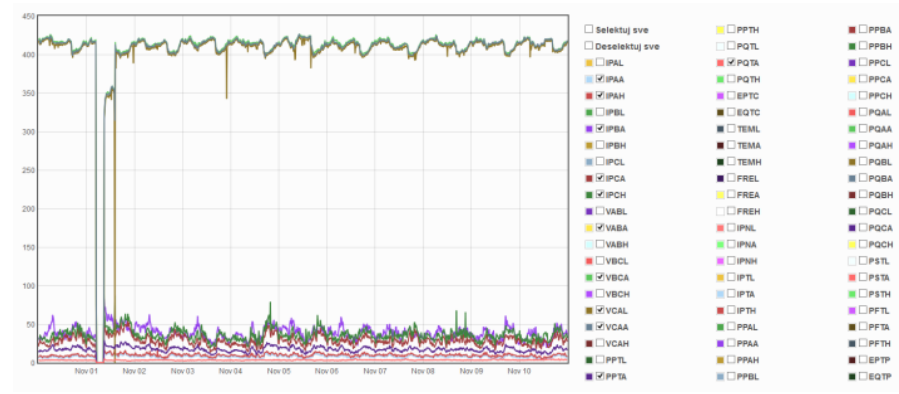

Fig. 6 Example of graphical reports for history measurement data 
The central part of the Web portal provides tools for generating different type of reports for the collected measurements data. At this point of application developments four types of reports are supported:

- History reports - presents all measurement readings from a sensor on a chosen location for a specified time interval.

- Reports for failed communications - presents all failed communications from one location for a specific time interval. These reports provide detailed information about failed communications, including the reason for the communication failure and whether the DAS service managed to solve the problem automatically.

- Current measurements - shows the latest measurement collected from the sensor on a specific location.

- Graphical report - graphical reports for history measurements reports for a specific location and time interval. Graphical report shows all values collected from one location or subset of these values can be defined (Fig. 6).

\section{Enterprise Information System}

Enterprise Information System (EIS) provides intelligent integration of information from a number of heterogeneous information sources [23]. Among other, EIS usually includes following data information sources: Geo-Information System (GIS), Automatic Meter Reading (AMR), Distribution Management System (DMS), Supervisory Control and Data Acquisition (SCADA), Billing System, and others. Each of them has its own database but they all share their information in order to create new relations between different pieces of data and provide new knowledge and information about an electric power grid. They communicate with each other through an Enterprise Service Bus combined with SOA techniques in order to give the best results. The new knowledge, information and integrated data are stored in the central Data warehouse.

Information from all services and systems that are part of the electrical utility company enterprise environment is stored in the same data warehouse. This includes both enterprise information system and measurements from electrical substations collected by DAS. This data is further available for analysis, processing and presentation to the users of EIS.

\section{COMMUNICATION RELIABILITY}

After having measuring devices deployed in the field and set up and connected, it is possible to collect measurements data to do various kinds of analyses. Securing reliable measurements transfer from measurement devices to the data centre is the most important goal that DAS has to provide. Reliability of communication in this system depends mostly on quality of the GSM $3 \mathrm{G}$ signal. Electrical substations equipped with measuring devices are located in different locations with different quality of GSM 3G or the signal quality can vary at the same location during the day. Low signal levels result in poor system performance, slow response times and reliability issues. This may result in data not being transmitted, irregular polling success or complete lack of connection. Any unsuccessful communication attempt is recorded by the service in failed communications log. In this way, the system keeps track of all data that is not transferred to the measurements database. 


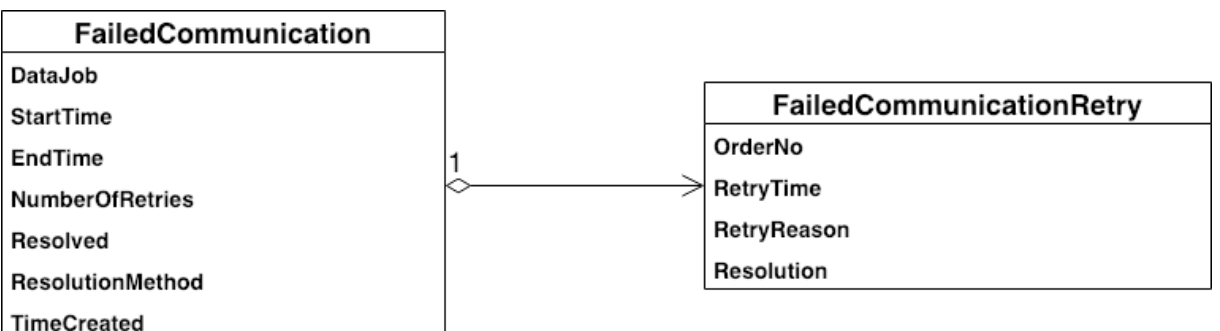

Fig. 7 Failed communications log simplified data model

To resolve the missing data problem, the system operator can try manually to read the missing data from the measuring device or, in the end, can physically visit the location of electrical substation and retrieve the data. This is a very expensive and impractical solution. For this purpose, the system implements several techniques which try to recover failed communication attempts.

All implemented techniques use data from the failed communications log. The simplified data model of this log is shown in Fig. 7. Every FailedCommunication object corresponds to a specific unsuccessful communication attempt. It contains detailed info about unsuccessful communication attempts:

- DataJob - Reference to a failed DAS communication job;

- StartTime and EndTime - In case of current measurements, only StartTime field is valid and define a point in time when DAS tried to read current conditions from measurement device. In case of history measurements, both fields are valid and define period of time for which DAS tried to obtain measurements data;

- NumberOfRetries - how many times system tried to retryfailed communication;

- Resolved - Status if failed communication is resolved or not;

- ResolutionMethod - How system managed to resolve a failed communication (manually or using a specific recovery technique);

- TimeCreated - point in time when FailedCommunication object was created.

Every FailedCommunication object also provides information about different attempts to resolve a failed communication. Every attempt is represented in the system as FailedCommunicationRetry object. This object provide data about specific attempt to resolve a failed communication:

- OrderNo - order number of an attempt;

- RetryTime - point in time when an attempt is made;

- RetryReason - why an attempt is made (operator manually tried to recover a failed communication or as a result of some recovery technique);

- Resolution - Status of an attempt: successful or unsuccessful.

\section{Approach based on failed communication retries}

This approach is based on simple failed communications retries when certain conditions are fulfilled. At the moment system implements two basic rules for failed communication retries: 
- Immediate retry (Fig. 8) - When a failed communication is detected for the first time, the system is using a time slot until the next data poll is scheduled to retry a failed communication for $\mathrm{N}$ times ( $\mathrm{N}$ is configurable for every measurement device);

- Retry if successful (Fig. 9) - When successful communication is detected, the system checks if there are unresolved failed communications for that measurement device and then tries to retry $\mathrm{N}$ failed communications for that device and to poll missing historical measurements data ( $\mathrm{N}$ is configurable for every measurement device).

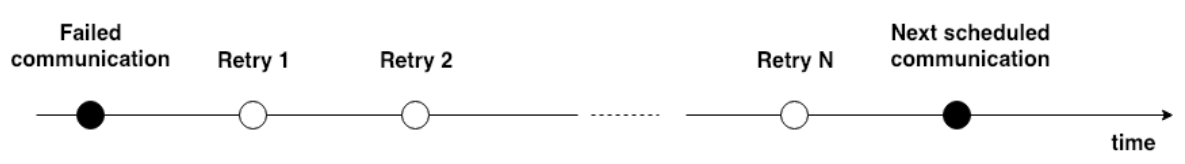

Fig. 8 Immediate retries technique

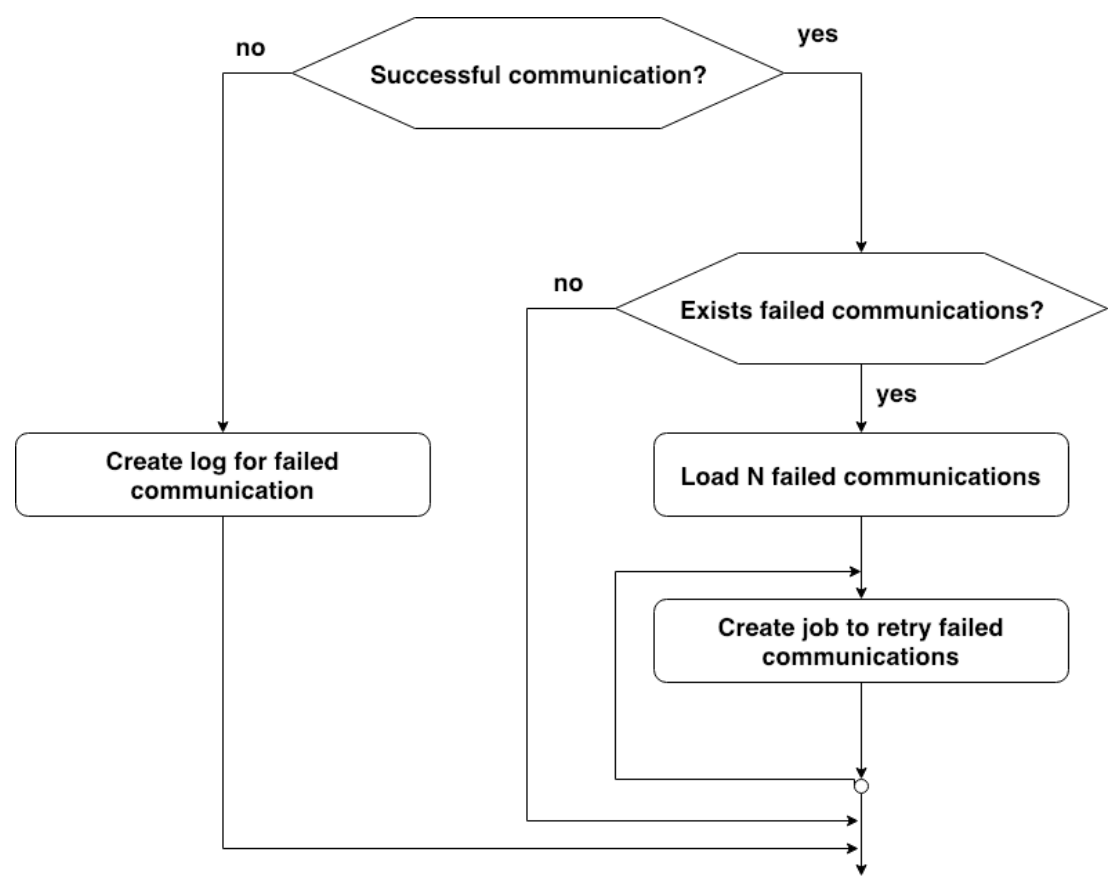

Fig. 9 Retry if successful technique

These simple techniques can resolve a large percent of all failed communications. The main disadvantage of this approach is a large number of unsuccessful retries that can cause poor performance of the system. This is the result of the fact that the existing system implementation treats all measurement devices in the same way regardless of the specifics of every measurement device. 


\section{Autonomous computing approach}

In order to increase communication reliability Data Gathering Service was extended with self-adaptation features in order to change the way how different measurement devices are treated during communication attempts or during failed communication recovery attempts. The basic goal was to decrease the number of failed communications and to decrease the number of unsuccessful retries. For this, an autonomous control loop approach is used. DAS is monitoring the failed communications log and in accordance with collected data, different adaptation steps (postpone failed communication retry, increase/decrease number of retries, etc.) can be triggered in correlation with geographical locations of the measurement device or time of day (if GSM 3G signal vary during the day on a specific location) or some other parameter.

In order to extend the DAS service with self-adaption features we need to implement an autonomic manager. The autonomic manager is responsible for monitoring the managed component and applying any changes when adaptation needs to be triggered. In the context of the DAS service, the managed element is a scheduled data poll job. The adaptation process is achieved using the well-known feedback loop [25]. It consists of four phases - measurement of managed element parameters, and then according to the observation - eventual analysis, planning and execution of an adaptive change into the managed element (MAPE loop) (Fig. 10).

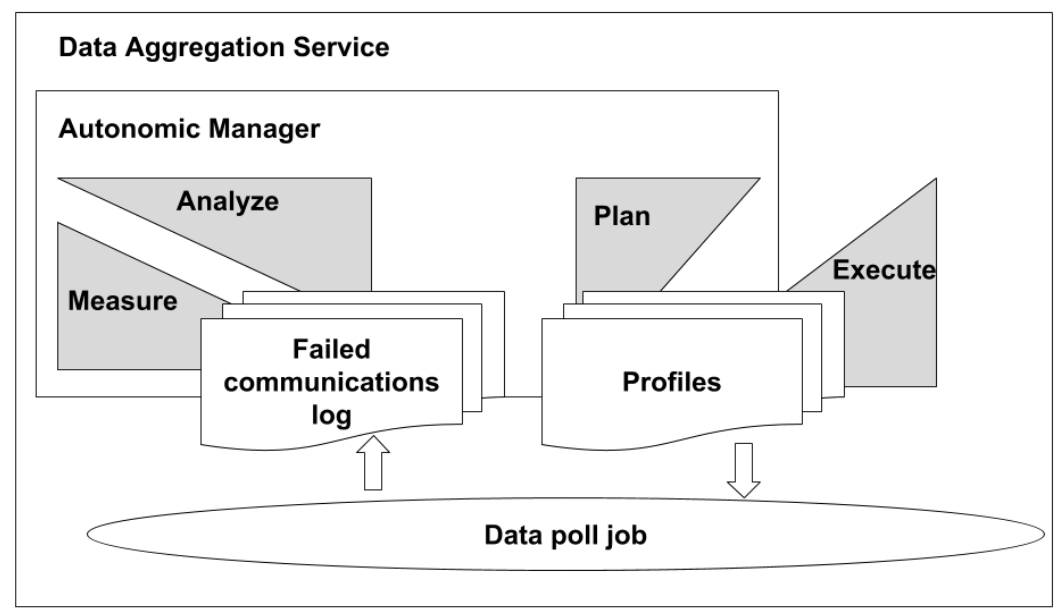

Fig. 10 Autonomic manager and self-adaptation control loop (MAPE loop)

In order to implement a self-adaptive control loop, the DAS service is using an independent data analyser. This is a separate monitoring job that constantly evaluates the failed communications log and prepares action plans for adaptation. This job runs daily and analyzes data from the failed communications log. During this phase, performances for different devices for different periods during a day are analyzed. The monitoring job tries to recognize patterns in failed communications. These patterns correspond with the geographical locations of measurement device (quality of GSM 3G signal), time of day (if GSM 3G signal vary during the day on specific location) or some other device specific parameter. 
As a result of these analyses different action plans for specific devices and their scheduled data poll jobs are generated. These action plans are created in a form of a communication profile that is attached to a specific data poll job. These profiles can contain various adaptation rules such as:

- Specify value for $N$ in immediate retry recovery rule - For some devices these is no point in running this step or we need to run this step with decreased number of retries. In this way we can significantly decrease the number of unsuccessful retries;

- Increase connection timeout - In case of deteriorated communication conditions, more time to transfer measurements data is needed and connection timeouts during data transfer should be avoided;

- Transfer measurements data in smaller chunks - When increased communication timeout are not enough. In these cases, measurements data should be transferred in smaller chunks: read different values from measurement devices in separate requests for current measurements or read measurement data points in smaller chunks for history measurements or combine both approaches;

- Postpone data poll jobs - this rule is important for polling history measurements. In some periods during a day, communication conditions can be deteriorated in a way that there is no point in trying to read data. In this case, data poll job should be postponed until there are acceptable communication conditions.

The DAS service is using created profiles in a data poll job execution phase. During job execution, the DAS service uses an assigned profile to define the context of a data poll job. This way, the DAS service can execute the data poll job in accordance to an assigned adaptation plan.

\section{CONCLUSIONS}

As mentioned previously, the LV distribution network monitoring system described in this paper was developed for the Electric Power Industry of Serbia (EPS) electricity distribution company. In this phase, the system is primarily used for monitoring operational parameters of middle voltage to low voltage electrical substations. At the moment, there are two large deployments of the monitoring system:

- EPS regional centre Belgrade with approximately 300 middle voltage to low voltage electrical substations covered and

- EPS regional centre Velika Plana with approximately 100 middle voltage to low voltage electrical substations covered.

Both deployments are in production for almost three years. These deployments cover two different use cases: Belgrade as a large urban area and Velika Plana as small town with adjoining rural settlements. As already stated, electrical substations equipped with measuring devices are located in different locations with different quality of GSM 3G or the signal quality can vary at the same location during the day. Also, there is a large difference of GSM $3 \mathrm{G}$ signal quality between urban and rural areas. Low signal levels result in poor system performance, slow response times and reliability issues.

In order to cope with the communication reliability, system was extended with algorithms for an automatic recovery of a failed communication request. When a failed communication is detected, the implemented system use communication retries in order to 
obtain missing measurement data. This approach can resolve a significant percent of failed communications, but also a large number of unsuccessful retries can be generated causing poor performance of the system.

In order to avoid these problems, the system was extended with an autonomic manager. This autonomic manager runs self-adaptive control loop and monitors behaviour of different groups of measurement devices. This monitoring is based on the analysis of collected failed communications data. Using this analysis, autonomic manager can create different profiles for different groups of measurement devices in accordance with their location and the quality of the GSM 3G signal on that location. Using this approach, a large number of failed communications is avoided, and number of unsuccessful failed communication retries is significantly decreased.

\section{REFERENCES}

[1] S.M. Amin, B.F. Wollenberg, Toward a smart grid: power delivery for the 21 st century. IEEE power and energy magazine, 3(5), pp.34-41, 2005. [Online]. Available: http://dx.doi.org/10.1109/MPAE.2005.1507024

[2] J. D. Taft, A. S. Becker-Dippmann, The Emerging Interdependence of the Electric Power Grid \& Information and Communication Technology (No. PNNL--24643). Pacific Northwest National Laboratory (PNNL), Richland, WA (United States), 2015.

[3] P. Siano, Demand response and smart grids - A survey. Renewable and Sustainable Energy Reviews, 30, pp.461-478, 2014. [Online]. Available: https://doi.org/10.1016/j.rser.2013.10.022

[4] B. Pfeiffer, P. Mulder, Explaining the diffusion of renewable energy technology in developing countries, Energy Economics, vol. 40, pp. 285-296, 2013. [Online]. Available: https://doi.org/10.1016/j.eneco.2013.07.005

[5] D. Della Giustina, A. Dedè, G. Invernizzi, D. P. Valle, F. Franzoni, A. Pegoiani, L. Cremaschini, Smart Grid Automation Based on IEC 61850: An Experimental Characterization, IEEE Transactions on Instrumentation and Measurement, vol.64, no.8, pp.2055,2063, 2015. [Online]. Available: https://doi.org/10.1109/TIM.2015.2415131

[6] S. Jamali, A. Bahmanyar, E. Bompard, Fault location method for distribution networks using smart meters, Measurement, vol. 102, pp. 150-157, 2017. [Online]. Available: https://doi.org/10.1016/j.measurement. 2017.02.008

[7] J. Leiva, A. Palacios, J. A. Aguado, Smart metering trends, implications and necessities: A policy review, Renewable and Sustainable Energy Reviews, vol. 55, pp. 227-233, 2016. [Online]. Available: https://doi.org/10.1016/j.rser.2015.11.002

[8] A. Barbato, A. Capone, Optimization models and methods for demand-side management of residential users: A survey, Energies, vol. 7, no. 9, pp. 5787-5824, 2014. [Online]. Available: https://doi.org/10.3390/ en7095787

[9] A. Grilo, L. Buttyan, J. Gonçalves, C. A. Fortunato, Wireless Sensor and Actuator Network for Improving the Electrical Power Grid Dependability, In Proceedings of the 8th EURO-NGI Conference on Next Generation Internet (NGI), Karlskrona, Sweden, 25-27 June 2012; pp. 71-78, 2012. [Online]. Available: https://doi.org/10.1109/NGI.2012.6252167

[10] R. Leon, A. Vittal, G. Manimaran, Application of sensor network for secure electric energy infrastructure, IEEE Trans. Power Delivery vol. 22, pp. 1021-1028, 2007. [Online]. Available: https://doi.org/10.1109/TPWRD. 2006.886797

[11] N. Dahal, V. M. Mohan, S. S. Durbha, A. K. Srivastava, R. L. King, N. H. Younan, N. N. Schulz, Wide area monitoring using Common Information Model and Sensor Web, In Proceedings of the Power Systems Conference and Exposition, Seattle, WA, USA, 15-18 March 2009, pp. 1-7, 2009. [Online]. Available: http://dx.doi.org/10.1109/PSCE.2009.4840207

[12] A. Biem, E. Bouillet, H. Feng, A. Ranganathan, A. Riabov, O. Verscheure, H. N. Koutsopoulos, M. Rahmani, B. Güç, Real-Time Traffic Information Management using Stream Computing, IEEE Data Eng. Bull., 33(2), pp.64$68,2010$.

[13] L. Neumeyer, B. Robbins, A. Nair, A. Kesari, S4: Distributed stream computing platform, 2010 IEEE International Conference on Data Mining Workshops, pp. 170-177, IEEE, 2010. [Online]. Available: https://doi.org/10.1109/ICDMW.2010.172 
[14] P. D. Diamantoulakis, V. M. Kapinas, G. K. Karagiannidis, Big data analytics for dynamic energy management in smart grids. Big Data Research, 2(3), pp.94-101, 2015. [Online]. Available: https://doi.org/ 10.1016/j.bdr.2015.03.003

[15] K. Sornalakshm, G. Vadivu, A Survey on Realtime Analytics Framework for Smart Grid Energy Management, In International Journal of Innovative Research in Science, Engineering and Technology, Vol. 4, Issue 3, pp. 1054-1058, ISSN(Online) : 2319-8753, 2015.

[16] M. Couceiro, R. Ferrando, D. Manzano, L. Lafuente, Stream analytics for utilities. Predicting power supply and demand in a smart grid, In Cognitive Information Processing (CIP), 2012 3rd International Workshop on (pp. 1-6). IEEE, 2012. [Online]. Available: https://doi.org/10.1109/CIP.2012.6232904

[17] The Right Big Data Technology for Smart Grid - Distributed Stream Computing [Online], Available> https://www.accenture.com/us-en/blogs/blogs-the-right-big-data-technology-for-smart-grid-distributedstream-computing, [Accessed on March 2019].

[18] V. Braberman, N. D'Ippolito, J. Kramer, D. Sykes, S. Uchitel, Morph: A reference architecture for configuration and behaviour self-adaptation, In: Proceedings of the 1st International Workshop on Control Theory for Software Engineering. ACM, pp. 9-16, 2015. [Online]. Available: https://doi.org/10. $1145 / 2804337.2804339$

[19] D. Garlan, S. W. Cheng, A. C. Huang, B. Schmerl, P. Steenkiste, Rainbow: Architecture-based selfadaptation with reusable infrastructure, Computer, 37(10), pp. 46-54, 2004. [Online]. Available: https://doi.org/10.1109/MC.2004.175

[20] S. W. Cheng, D. Garlan, B. Schmerl, Evaluating the effectiveness of the rainbow self-adaptive system, In: Software Engineering for Adaptive and Self-Managing Systems, SEAMS'09. ICSE Workshop on. IEEE, pp. 132-141, 2009. [Online]. Available: https://doi.org/10.1109/SEAMS.2009.5069082

[21] J. O. Kephart, D. M. Chess, The vision of autonomic computing, Computer 36(1), pp. 41-50, 2003. [Online]. Available: https://doi.org/10.1109/MC.2003.1160055

[22] Y. Brun, G. D. Serugendo, C. Gacek, H. Giese, H. Kienle, M. Litoiu, H. Müller, M. Pezzè, M. Shaw, Engineering Self-Adaptive Systems through Feedback Loops, In Software Engineering for Self-Adaptive Systems, LNCS, Vol. 5525. Springer-Verlag, pp. 48-70, 2009. [Online]. Available: https://doi.org/10. 1007/978-3-642-02161-9_3

[23] L. Stoimenov, A. Stanimirovic, A. Krstic, N. Davidovic, M. Bogdanovic, D. Nikolic, GinisED Enterprise GIS - Framework for the utility of the future, 21st International Conference on Electricity Distribution, Frankfurt, June 6-9, paper 1223, 2011.

[24] Netico NTPM 100/200 Energy Management Sensor. [Online]. Available: http://netico-group.com/ NTPM-100200, [Accessed on March 2019]

[25] S. Gokhale, Architecture-Based Software Reliability Analysis: Overview and Limitations, IEEE Transactions on Dependable Security Computing, vol. 4, no. 1, pp. 32-40, 2007. [Online]. Available: https://doi.org/10.1109/TDSC.2007.4 